\title{
Descripción de factores clínicos asociados a la torsión testicular
}

\section{Description of Clinical Factors Associated with Testicular Torsion}

\author{
José Nicolás Fernández Bonilla ${ }^{1}$ Mauricio Medina-Rico ${ }^{2}$ Luisa Fernanda Rincón ${ }^{3}$ \\ Jaime Francisco Pérez Niño ${ }^{4}$
}
${ }^{1}$ Departamento de Urología, Hospital Universitario San Ignacio, Profesor Facultad de Medicina, Pontificia Universidad Javeriana, Bogotá, Colombia
2 Médico-Cirujano, Facultad de Medicina, Hospital Universitario San Ignacio, Pontificia Universidad Javeriana, Bogotá, Colombia
3 Interno XII Semestre, Facultad de Medicina, Hospital Universitario San Ignacio, Pontificia Universidad Javeriana, Bogotá, Colombia
4 Departamento de Urología, Hospital Universitario San Ignacio, Profesor Facultad de Medicina, Pontificia Universidad Javeriana, Bogotá, Colombia

\author{
Address for correspondence Mauricio Medina Rico, MD, Hospital \\ Universitario San Ignacio, Cra 7 \# 40 - 62, Departamnto de \\ Epidemiología Clínica y bioestadística, 2do piso, Pontificia \\ Universidad Javeriana, Bogotá, Colombia \\ (e-mail: medina.mauricio@javeriana.edu.co).
}

Urol Colomb 2018;27:167-173.

\section{Resumen}

\section{Palabras Clave}

- escroto agudo

- dolor agudo

- torsión testicular

- tiempo real

- diagnóstico

- manejo
Introducción La torsión testicular es una urgencia urológica que afecta 4,5 de cada 100.000 hombres menores de 25 años. Requiere un diagnóstico oportuno y una intervención en las primeras 4 a 6 horas para evitar la pérdida irreversible del parénquima testicular.

Objetivo Describir los signos y síntomas más relevantes en el diagnóstico de torsión testicular.

Materiales y Métodos Se recogió información de pacientes que consultaron por urgencias entre enero de 2010 y enero de 2015. De las historias clínicas se extrajeron: edad, lateralidad, síntomas, hallazgos al examen físico, intervención y hallazgos intraoperatorios. Las variables fueron evaluadas mediante análisis bivariado.

Resultados Un total de 117 pacientes manifestaron como determinantes diagnósticos de torsión testicular: La edad entre 10 y 18 años, $67 \%$ de los casos $(p=0,0001)$. Al examen físico el tamaño del hemiescroto, la posición y orientación del testículo se presentaron respectivamente: $85 \%$ aumentado $(p=0,046), 60 \%$ ascendido $(p=0,048)$ y $67 \%$ horizontalizado $(p=0,004)$. El dolor "pulsatil" fue el más frecuente, descrito en un $63,4 \%$ de los $\operatorname{casos}(p=0,028)$. Los que consultaron en las primeras 12 horas tuvieron mejor desenlace $63 \%(p=0,0001)$ al igual que los intervenidos en las primeras 6 horas $80 \%(p=0,049)$.

Conclusiones Los hallazgos muestran tener relación con la literatura. El reflejo cremastérico y la presentación de orquialgias previas, deben ser evaluadas con más profundidad. Es importante educar al médico general y al paciente en el diagnóstico de received

June 13, 2016

accepted

February 8, 2018

published online

April 24, 2018
DOI https://doi.org/

10.1055/s-0038-1645841.

ISSN 0120-789X.

eISSN 2027-0119.
Copyright ( 2018, Sociedad Colombiana License terms de Urología. Publicado por Thieme Revinter Publicações Ltda., Rio de Janeiro, Brazil. Todos los derechos reservados.

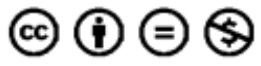


la torsión testicular, para mejorar el tiempo de atención y el lapso hasta la intervención y así mejorar el pronóstico del paciente.

\begin{abstract}
Introduction Testicular torsion is a urological emergency that affects 4.5 out of 100,000 men under 25 years. It requires prompt diagnosis and intervention within the first 4 to 6 hours to avoid irreversible loss of testicular parenchyma.

Objective To describe the most important signs and symptoms in the diagnosis of testicular torsion.

Materials and Methods We extracted information from the clinical records of patients who consulted to the emergency department between January 2010 and January 2015. The analyzed variables were: age, laterality, related symptoms, physical examination findings and intraoperative findings. The variables were evaluated by bivariate analysis.

Results A total of 117 patients presented with testicular torsion: age range was between 10 and 18 years, $67 \%$ of cases $(p=0.0001)$. In the physical exam increased hemiscrotum size was present in $85 \%(p=0.046)$, an ascended position was seen in $60 \%(p=0.048)$ and $67 \%$ were horizontalized $(p=0.004)$. Pain described as "pulsatile" was the most common type in $63.4 \%$ of the cases $(p=0.028)$. Those

Keywords

- acute scrotum

- acute pain

- testicular torsion

- real time

- diagnosis

- management who consulted within the first 12 hours had a better outcome $(p=0.0001)$, as well as those intervened within the first 6 hours $(p=0.049)$.

Conclusions Clinical presentation alone can make the diagnosis of testicular torsion. Cremasteric reflex and presentation of previous episodes of orchyialgia should be evaluated more thoroughly in future studies. It is important to educate the general practitioners and the patient in the diagnosis of testicular torsion to improve attention and intervention time to improve patient prognosis.
\end{abstract}

\section{Introducción}

El escroto agudo es una de las pocas condiciones urológicas que requiere intervención inmediata. ${ }^{1}$ La principal causa de escroto agudo es la torsión testicular y afecta a 4,5 de cada 100.000 hombres menores de 25 años. $^{2}$ Se presenta por una predisposición dada por un defecto congénito de la inserción del testículo al escroto mediante un gubernaculum testis largo y/o con una implantación posterior que le permite al testículo girar libremente sobre su propio eje. ${ }^{3}$

Los signos y síntomas más frecuentes en el diagnóstico de torsión testicular son: dolor escrotal severo con una presentación entre $82 \%$ a $100 \%$ de los casos. ${ }^{4}$ Progresión del dolor en las primeras 24 horas $^{5}$ y signos inflamatorios locales (eritema, calor, edema), están presentes en tres cuartas partes de los casos. ${ }^{6}$ Las náuseas con o sin presencia de emesis están presentes en más de la mitad de los casos, al igual que el signo de Gouverneur positivo. ${ }^{4}$ La presencia de orquialgias previas son variables muy importantes a ser evaluadas. ${ }^{1,3,7,8}$

Sin embargo, pocos estudios han realizado un análisis estadístico para determinar los valores pronósticos de cada uno de los signos y síntomas ya mencionados. Boettcher y col., determinó un valor predictivo positivo para las náuseas y la emesis entre el $74 \%$ y el $96 \%$, seguido por la ausencia del reflejo cremastérico en $43 \%$ y $83 \%$, y el cambio de orientación del testículo del $50 \%{ }^{6}$
Srinivasan y col., mediante análisis multivariado, encontró que: los cambios escrotales, la ausencia del reflejo cremastérico, las náuseas y la emesis al presentarse los tres o por el contrario, estar ausentes, aumentan de manera considerable sus valores predictivos, tanto negativos como positivos. ${ }^{9}$ La ausencia de un solo hallazgo no debería ser suficiente para descartar la torsión. 5,9

La intervención quirúrgica de la torsión testicular debe realizarse idealmente en las primeras 4-6 horas, para tener un éxito de salvar el testículo mayor al $90 \%$, siendo en su mayoría irreversible después de 24 horas. ${ }^{4,8,10-12}$ Las intervenciones a realizar en esos pacientes depende de la gravedad del cuadro, siendo desde la destorsión y recuperación del testículo, hasta la orquiectomía. ${ }^{11,13}$

El objetivo del estudio es identificar los signos y síntomas más relevantes en el diagnóstico de torsión testicular, así como determinar cuál es el tiempo ideal de intervención para lograr un abordaje más efectivo del paciente con torsión testicular.

\section{Materiales y Métodos}

Se realizó una recolección de datos de pacientes que consultaron al servicio de urgencias entre enero de $2010 \mathrm{y}$ enero de 2015. Los criterios de inclusión fueron: Edad de 25 años o menor. Consulta por orquialgia sugestiva de torsión testicular por historia clínica y examen físico el cual debía ser 
realizado por urólogo. Confirmación diagnóstica de la torsión testicular mediante la exploración quirúrgica.

Se excluyeron aquellos pacientes que hubieran tenido antecedente de trauma escrotal asociado al cuadro de escroto agudo, aquellos cuya patología resultara positiva para neoplasia testicular o que durante la intervención no se observaran hallazgos sugestivos de torsión testicular.

De las historias clínicas se extrajo información sobre: localización, tipo de dolor descrito, irradiación del dolor, historia de orquialgia previa, síntomas asociados como: emesis, nauseas, hallazgos al examen físico (aspecto del escroto, elevación del testículo o signo de Gouverneur y la presencia de reflejo cremastérico), tiempo de evolución desde el inicio del dolor a la valoración, tiempo de la valoración a la intervención, hallazgos intraoperatorios. En caso de haber sido llevados a orquiectomía, el resultado de la patología.

Mediante el programa Stata se realizó un análisis bivariado de carácter regresivo, con el propósito de encontrar posibles asociaciones entre: edad de los pacientes, tipo de dolor (punzante, opresivo o pulsatil), lateralidad del dolor (izquierdo o derecho), hallazgos al examen físico (Signos inflamatorios locales, posición, orientación del testículo y reflejo cremastérico), sintomatología (emesis, irradiación y episodios previos) y el diagnóstico (torsión de hidátide, torsión testicular). Igualmente se quiso observar si las variables ya mencionadas además de: el tiempo de evolución desde el inicio del dolor hasta su atención por urgencias, y tiempo desde su atención por urgencias hasta su ingreso a salas de cirugía, pueden ser determinantes en el pronóstico del paciente, por lo que se analizaron respecto a la intervención quirúrgica (orquiectomía o recuperación de función testicular).

\section{Resultados}

Se revisó un total de 117 historias clínicas, de las cuales $11 \%$ resultaron con diagnóstico de torsión de hidátide, 49\% tuvieron torsión testicular con recuperación de la función testicular posterior a la distorsión y $40 \%$ se llevaron a orquiectomía. Ningún paciente correspondió a un cuadro de orquiepidimitis, epidimitis o púrpura de Henoch-Schonlein.

Al realizar el análisis multivariado para determinar la asociación entre las variables y el diagnóstico de torsión testicular, se encontró una diferencia estadísticamente significativa para la edad en relación con la torsión testicular teniendo una mayor manifestación entre los $10 \mathrm{y}$ 18 años (67\%), y siendo rara su presentación antes de los 10 años de $3 \%(p=0,0001)$. De los pacientes con torsión de hidátide, se evidenció una presentación infrecuente después de los 18 años (7\%) (-Tabla 1).

Para el diagnóstico de torsión testicular los hallazgos al examen físico que se encontraron con mayor frecuencia fueron: Aumento del tamaño del hemiescroto afectado, evidenciando ser un hallazgo significativo en el $85 \%$ de los casos $(p=0,046)$. La alteración tanto de la posición como la orientación del testículo mostraron ser determinantes en el diagnóstico de torsión testicular presentándose en un $60 \%$ ascendido $(p=0,048)$ y $67 \%$ horizontalizado $(p=0,004)$.
Respecto a la presencia o ausencia del reflejo cremastérico, el cambio de coloración en el escroto y la lateralidad del cuadro no mostraron diferencias estadísticamente significativas para el diagnóstico de torsión testicular. En cuanto a la sintomatología de la torsión testicular, el tipo de dolor caracterizado como "pulsátil", fue el más frecuente presentándose en el $63,4 \%$ de los casos $(p=0,028)$, y la ausencia de emesis $56 \%(p=0,014)$. La irradiación del dolor aL abdomen pese a observarse en el 35,9\% de los casos, o la presencia de oquialgias previas en un $36,2 \%$, no fueron estadísticamente significativas para el diagnóstico de torsión testicular ( $\mathbf{- T a b l a ~} \mathbf{1})$.

Del análisis para determinar el pronóstico del paciente, las únicas variables que tuvieron un valor estadísticamente significativo fueron: el tiempo desde el comienzo del dolor hasta la atención por urgencias en la que la mayoría de los pacientes atendidos en las primeras 12 horas tuvieron más probabilidad de que recuperaran la función testicular $63 \%$ $(p=0,0001)$ y el tiempo desde la atención hasta la realización de la intervención con mejor probabilidad de recuperación testicular en aquellos intervenidos en las primeras 6 horas $80 \%(p=0,049)$. Así mismo la presencia de episodios de orquialgias previas pareció ser determinante de un buen pronóstico presentándose en el 48,94\% de los casos de las personas que tuvieron preservación testicular $(p=0,035)$. Ningún otro signo, síntoma, o hallazgo al examen físico mostró tener valor estadísticamente significativo para predecir la viabilidad del testículo (- Tabla 2).

El tiempo medio desde el comienzo de los síntomas hasta la primera consulta por urgencias fue de 37 horas; no obstante, al hacer este cálculo considerando las personas que consultaron en las primeras 24 horas, la media fue de 10,5 horas. De la misma manera, el tiempo promedio de espera entre la consulta y la intervención quirúrgica fue de 16 horas. Sin embargo, al tomar la cifra de los pacientes operados en las primeras 12 horas posteriores a la consulta, el promedio fue de 4,75 horas.

Teniendo en cuenta los hallazgos anteriores, se encontró una relación entre el tiempo de inicio de los síntomas y el tiempo hasta la realización de la cirugía. El resultado fue que el $72,6 \%$ de la población estudiada fue intervenida en las primeras 6 horas. Dicha proporción de pacientes intervenidos fue del 95\% considerando solamente los pacientes que consultaron en las primeras 12 horas desde el inicio de los síntomas. Por el contrario, un $49 \%$ de los pacientes que consultaron después de las 24 horas de inicio del dolor serían intervenidos en las primeras 6 horas (Ver - Tabla 3).

\section{Discusión}

Los resultados encontrados evidenciaron similitud con lo descrito en otros estudios, identificando como principales factores a tener en cuenta: La edad del sujeto siendo más frecuente durante y después de adolescencia, la presencia de dolor intenso de descripción pulsátil, el aumento del tamaño del hemiescroto, la horizontalización y el ascenso del testículo afectado en el examen físico. ${ }^{6}$ Así mismo, los tiempos desde el comienzo de los síntomas hasta la 
Tabla 1 Diagnóstico de torsión testicular y asociación con: signos, síntomas, y hallazgos al examen físico

\begin{tabular}{|c|c|c|c|c|c|c|c|}
\hline & \multicolumn{2}{|c|}{ Torsión de Hidátide } & \multicolumn{2}{|c|}{ Torsión Testicular } & \multicolumn{2}{|l|}{ Total } & \multirow[b]{2}{*}{$P$} \\
\hline & Frecuencia & $\%$ & Frecuencia & $\%$ & Frecuencia & $\%$ & \\
\hline \multicolumn{8}{|l|}{ Edad } \\
\hline$<$ de10 años & 5 & $38,46 \%$ & 3 & $2,88 \%$ & 8 & $6,84 \%$ & \multirow[t]{3}{*}{0,0001} \\
\hline entre 10 y 18 años & 7 & $53,85 \%$ & 70 & $67,31 \%$ & 77 & $65,81 \%$ & \\
\hline$>$ de 18 años & 1 & $7,69 \%$ & 31 & $29,81 \%$ & 32 & $27,35 \%$ & \\
\hline \multicolumn{8}{|l|}{ Tamaño } \\
\hline Aumentado & 8 & $61,54 \%$ & 89 & $85,58 \%$ & 97 & $82,91 \%$ & \multirow[t]{2}{*}{0,046} \\
\hline No descrito & 5 & $38,46 \%$ & 15 & $14,42 \%$ & 20 & $17,09 \%$ & \\
\hline \multicolumn{8}{|l|}{ Color } \\
\hline Eritema & 6 & $46,15 \%$ & 23 & $22,12 \%$ & 29 & $24,79 \%$ & \multirow[t]{2}{*}{0,066} \\
\hline Sin cambios & 7 & $53,85 \%$ & 81 & $77,88 \%$ & 88 & $75,21 \%$ & \\
\hline \multicolumn{8}{|l|}{ Posición } \\
\hline Ascendido & 4 & $30,77 \%$ & 62 & $59,62 \%$ & 66 & $56,41 \%$ & \multirow[t]{2}{*}{0,048} \\
\hline En bolsa escrotal & 9 & $69,23 \%$ & 42 & $40,38 \%$ & 51 & $43,59 \%$ & \\
\hline \multicolumn{8}{|l|}{ Orientación } \\
\hline Anatómica & 10 & $76,92 \%$ & 34 & $32,69 \%$ & 44 & $37,61 \%$ & \multirow[t]{2}{*}{0,004} \\
\hline Horizontalizado & 3 & $23,08 \%$ & 70 & $67,31 \%$ & 73 & $62,39 \%$ & \\
\hline \multicolumn{8}{|l|}{ Refejo cremastérico } \\
\hline Ausente & 5 & $38,46 \%$ & 70 & $67,31 \%$ & 75 & $64,10 \%$ & \multirow[t]{3}{*}{0,065} \\
\hline No descrito & 4 & $30,77 \%$ & 12 & $11,54 \%$ & 16 & $13,68 \%$ & \\
\hline Presente & 4 & $30,77 \%$ & 22 & $21,15 \%$ & 26 & $22,22 \%$ & \\
\hline \multicolumn{8}{|l|}{ Lateralidad } \\
\hline Derecho & 4 & $30,77 \%$ & 50 & $48,08 \%$ & 54 & $46,15 \%$ & \multirow[t]{2}{*}{0,238} \\
\hline Izquierdo & 9 & $69,23 \%$ & 54 & $51,92 \%$ & 63 & $53,85 \%$ & \\
\hline \multicolumn{8}{|l|}{ Tipo de dolor } \\
\hline No específico & 2 & $15,38 \%$ & 1 & $0,94 \%$ & 3 & $2,56 \%$ & \multirow[t]{4}{*}{0,028} \\
\hline Opresivo & 3 & $23,08 \%$ & 31 & $29,81 \%$ & 34 & $29,06 \%$ & \\
\hline Pulsátil & 6 & $46,15 \%$ & 66 & $63,46 \%$ & 72 & $61,54 \%$ & \\
\hline Punzada & 2 & $15,38 \%$ & 6 & $5,77 \%$ & 8 & $6,84 \%$ & \\
\hline \multicolumn{8}{|l|}{ Irradiación } \\
\hline No & 9 & $69,23 \%$ & 66 & $64,10 \%$ & 75 & $64,10 \%$ & \multirow[t]{2}{*}{0,768} \\
\hline Sí & 4 & $30,77 \%$ & 38 & $35,90 \%$ & 42 & $35,90 \%$ & \\
\hline \multicolumn{8}{|l|}{ Episodios previos } \\
\hline No & 5 & $38,46 \%$ & 28 & $26,92 \%$ & 33 & $28,21 \%$ & \multirow[t]{3}{*}{0,617} \\
\hline No se pregunta & 5 & $38,46 \%$ & 38 & $36,54 \%$ & 43 & $36,75 \%$ & \\
\hline Sí & 3 & $23,08 \%$ & 38 & $36,52 \%$ & 41 & $35,04 \%$ & \\
\hline \multicolumn{8}{|l|}{ Emesis } \\
\hline No & 12 & $92,31 \%$ & 58 & $55,77 \%$ & 70 & $59,83 \%$ & 0,014 \\
\hline Sí & 1 & $7,69 \%$ & 46 & $44,23 \%$ & 47 & $40,17 \%$ & \\
\hline
\end{tabular}

consulta y el lapso desde la consulta hasta la intervención, son los principales determinantes del pronóstico del paciente, hallazgo descrito por otros autores que describen de manera general, el tiempo desde el comienzo del dolor hasta la intervención como el principal factor para un buen pronóstico en esos pacientes. ${ }^{14}$
En cuanto al tiempo de atención y el lapso hasta la intervención, se encontró una relación donde a mayor demora del paciente por consultar, mayor demora hasta la realización de la intervención. Eso puede deberse a que pasado un tiempo se da por hecho la pérdida del testículo logrando disminuir de esa forma el carácter "urgente" que 
Tabla 2 Desenlace del paciente y asociación con: signos, síntomas, hallazgos al examen físico y tiempos de atención

\begin{tabular}{|c|c|c|c|c|c|c|c|}
\hline & \multicolumn{2}{|c|}{ Infarto Testicular } & \multicolumn{2}{|c|}{$\begin{array}{l}\text { Preservación Del } \\
\text { Testículo }\end{array}$} & \multicolumn{2}{|l|}{ Total } & \multirow[b]{2}{*}{$P$} \\
\hline & Frecuencia & $\%$ & Frecuencia & $\%$ & Frecuencia & $\%$ & \\
\hline \multicolumn{8}{|l|}{ Refejo cremastérico } \\
\hline Ausente & 33 & $70,21 \%$ & 42 & $60,00 \%$ & 75 & $64,10 \%$ & \multirow[t]{3}{*}{0,517} \\
\hline No descrito & 5 & $10,64 \%$ & 11 & $15,71 \%$ & 16 & $13,68 \%$ & \\
\hline Presente & 9 & $19,15 \%$ & 17 & $24,29 \%$ & 26 & $22,22 \%$ & \\
\hline \multicolumn{8}{|l|}{ Posición } \\
\hline Ascendido & 26 & $55,32 \%$ & 40 & $57,14 \%$ & 66 & $56,41 \%$ & \multirow[t]{2}{*}{0,845} \\
\hline En bolsa escrotal & 21 & $44,68 \%$ & 30 & $42,86 \%$ & 51 & $43,59 \%$ & \\
\hline \multicolumn{8}{|l|}{ Orientación } \\
\hline Anatómica & 15 & $31,91 \%$ & 29 & $41,43 \%$ & 44 & $37,61 \%$ & \multirow[t]{2}{*}{0,298} \\
\hline Horizontalizado & 32 & $68,09 \%$ & 41 & $58,57 \%$ & 73 & $62,39 \%$ & \\
\hline \multicolumn{8}{|l|}{ Color } \\
\hline Eritema & 16 & $33,33 \%$ & 13 & $18,84 \%$ & 29 & $24,79 \%$ & \multirow[t]{2}{*}{0,057} \\
\hline Sin cambios & 31 & $66,67 \%$ & 57 & $81,16 \%$ & 88 & $75,21 \%$ & \\
\hline \multicolumn{8}{|l|}{ Lateralidad } \\
\hline Derecho & 22 & $46,81 \%$ & 32 & $48,48 \%$ & 54 & $46,15 \%$ & \multirow[t]{2}{*}{0,485} \\
\hline Izquierdo & 25 & $53,19 \%$ & 34 & $51,52 \%$ & 63 & $53,85 \%$ & \\
\hline \multicolumn{8}{|l|}{ Tipo de dolor } \\
\hline No específico & 1 & $2,13 \%$ & 2 & $2,86 \%$ & 3 & $2,56 \%$ & \multirow[t]{4}{*}{0,298} \\
\hline Opresivo & 18 & $38,30 \%$ & 16 & $22,86 \%$ & 34 & $29,06 \%$ & \\
\hline Pulsátil & 26 & $55,32 \%$ & 46 & $65,71 \%$ & 72 & $61,54 \%$ & \\
\hline Punzada & 2 & $4,26 \%$ & 6 & $8,57 \%$ & 8 & $6,84 \%$ & \\
\hline \multicolumn{8}{|l|}{ Irradiación } \\
\hline No & 33 & $70,21 \%$ & 42 & $60,00 \%$ & 75 & $64,10 \%$ & \multirow[t]{2}{*}{0,259} \\
\hline Sí & 14 & $29,79 \%$ & 28 & $40,00 \%$ & 42 & $35,90 \%$ & \\
\hline \multicolumn{8}{|l|}{ Episodios previos } \\
\hline No & 10 & $21,28 \%$ & 23 & $32,86 \%$ & 33 & $28,21 \%$ & \multirow[t]{3}{*}{0,035} \\
\hline No se pregunta & 14 & $29,79 \%$ & 29 & $41,43 \%$ & 43 & $36,75 \%$ & \\
\hline Sí & 23 & $48,94 \%$ & 18 & $25,71 \%$ & 41 & $35,04 \%$ & \\
\hline \multicolumn{8}{|l|}{ Emesis } \\
\hline No & 29 & $61,70 \%$ & 41 & $58,57 \%$ & 70 & $59,83 \%$ & \multirow[t]{2}{*}{0,735} \\
\hline Sí & 18 & $38,30 \%$ & 29 & $41,43 \%$ & 47 & $40,17 \%$ & \\
\hline \multicolumn{8}{|c|}{ Tiempo hasta la consulta } \\
\hline$<12$ horas & 12 & $25,53 \%$ & 44 & $62,86 \%$ & 56 & $47,86 \%$ & \multirow[t]{3}{*}{0,0001} \\
\hline Entre 12 y 24 horas & 10 & $21,28 \%$ & 14 & $20,00 \%$ & 24 & $20,51 \%$ & \\
\hline$>24$ horas & 25 & $53,19 \%$ & 12 & $17.14 \%$ & 37 & $31,62 \%$ & \\
\hline \multicolumn{8}{|c|}{ Tiempo hasta la cirugía } \\
\hline$<6$ horas & 29 & $61,70 \%$ & 56 & $80 \%$ & 85 & $72,65 \%$ & 0,049 \\
\hline Entre 6 y 12 horas & 8 & $17,02 \%$ & 9 & $12,86 \%$ & 18 & $14,53 \%$ & \\
\hline$>12$ horas & 10 & $21,28 \%$ & 5 & $7.14 \%$ & 15 & $12,82 \%$ & \\
\hline
\end{tabular}

implica la torsión testicular. No obstante, se encontraron algunos casos de reperfusión del tejido hasta después de 24 horas de inicio de los síntomas, haciendo evidente la necesidad de no desestimar la posibilidad de recuperación del testículo y con ello no retrasar la intervención de un paciente con sospecha de torsión testicular, a pesar de pasadas las horas de viabilidad descritas por la literatura.

Finalmente nuestro estudio evidencia como hallazgo interesante la presencia de orquialgias previas como posible factor pronóstico, el cual puede asociarse a la presencia de 
Tabla 3 Relación entre tiempo de atención y lapso hasta la intervención

\begin{tabular}{|c|c|c|c|c|c|c|c|}
\hline & \multicolumn{6}{|c|}{ Tiempo hasta la cirugía } & \multirow[b]{3}{*}{$P$} \\
\hline & \multicolumn{2}{|l|}{$<6$ horas } & \multicolumn{2}{|c|}{ Entre 6 y 12 horas } & \multicolumn{2}{|l|}{$>12$ horas } & \\
\hline & Frecuencia & $\%$ & Frecuencia & $\%$ & Frecuencia & $\%$ & \\
\hline \multicolumn{8}{|c|}{ Tiempo hasta la consulta } \\
\hline$<12$ horas & 53 & $94,64 \%$ & 3 & $5,36 \%$ & 0 & $0 \%$ & \multirow[t]{3}{*}{0,0001} \\
\hline Entre 12 y 24 horas & 14 & $58,33 \%$ & 5 & $20,83 \%$ & 5 & $20,83 \%$ & \\
\hline$>24$ horas & 18 & $48,65 \%$ & 9 & $24,32 \%$ & 10 & $27,03 \%$ & \\
\hline
\end{tabular}

cuadros de torsión testicular intermitentes debido a cuadros de destorsión involuntaria, ${ }^{15}$ por lo cual se podría suponer que esos pacientes pueden tener cuadros de destorsión parcial que permita algún grado de flujo sanguíneo al testículo que aumente el umbral de viabilidad del testículo, o porque la evaluación de esos pacientes fue más meticulosa y eso hizo más probable llevar al paciente a cirugía.

Como aportes a la práctica médica con los hallazgos de este estudio, se propone educar a médicos y especialistas sobre la importancia de atender con carácter prioritario a todos los pacientes que lleguen a los servicios de Urgencias con cuadros sugestivos de torsión testicular cuyo cuadro sintomático sea menor a 24 horas de evolución, principalmente en aquellos que refieran haber tenido orquialgias previas por la probabilidad de que el testículo aun pueda ser recuperado. Así mismo, se deberían extender propuestas de educación en escuelas durante la enseñanza de educación sexual, siendo ese un cuadro que se presenta en la juventud, o a familiares y pacientes cuando consultan a urología para que ellos sepan de la importancia de la atención en las primeras horas del dolor.

Una de las limitaciones del estudio radica en la ausencia de datos completos en la historia clínica del paciente. Al no describirse todos los posibles hallazgos, tal y como se evidencia en el caso de la presencia de orquialgias previas en donde en la tercera parte de los pacientes no se tiene ese dato. Igualmente, la presencia o ausencia del reflejo cremastérico que no se documenta en la quinta parte de los sujetos, lo que hubiera podido cambiar el resultado. Así mismo este estudio no indagó sobre los hallazgos ecográficos dado que desde su contemplación y considerando la práctica clínica diaria, un grupo considerable de pacientes son intervenidos antes de la realización de la ecografía, dados los hallazgos clínicos y el tiempo que se requiere. No obstante, tras nuestros hallazgos valdría la pena para futuros estudios, evaluar el tiempo que la realización del Doppler conlleva, para determinar su utilidad en aquellos pacientes cuyo cuadro sintomático sea dudoso y se encuentre en un tiempo que permita la posibilidad de la realización del Doppler para descartar otras condiciones con cuadros similares como la orquiepidimitis, epidimitis o púrpura de Henoch-Schonlein.

\section{Conclusiones}

El diagnóstico e intervención temprana de la torsión testicular, siguen siendo los pilares para la recuperación de la función testicular del paciente que consulta por torsión testicular.

Nuestro estudio sirve como base para la educación del paciente acerca de la torsión testicular en cuanto a la importancia de la atención temprana del mismo, así como para el entrenamiento del personal médico para la identificación efectiva de la torsión testicular en el sujeto que ingresa al servicio de urgencias con cuadro de escroto agudo, lo cual puede influir en el lapso entre diagnóstico e intervención.

Finalmente, este artículo puede dar origen a futuros estudios con muestras poblacionales más grandes de pacientes que puedan ofrecer información adicional de algunas de las variables que se analizaron y no fueron concluyentes, así como la realización de estudios dirigidos a la identificación de los factores que pueden retrasar el diagnóstico e intervención del paciente con torsión testicular y de cómo modificarlos.

Responsabilidades éticas

Protección de personas y animales. Los autores declaran que para esta investigación, no se han realizado experimentos en seres humanos ni en animales.

Confidencialidad de los datos. Los autores declaran que han seguido los protocolos de su centro de trabajo sobre la publicación de datos de pacientes.

Derecho a la privacidad y consentimiento informado. Los autores declaran que en este artículo no aparecen datos de pacientes.

Conflictos de Interés

Los autores declaran no tener ningún tipo de conflicto de interés en relación a este estudio.

\section{Bibliografía}

1 Srinath H. Acute scrotal pain. Aust Fam Physician 2013;42(11): 790-792

2 Mergers H, Feb VW. Harvard University; Testicular torsion and risk factors for orchiectomy discussed. Resumen 2006;762:1-3

3 Gordhan CG, Sadeghi-Nejad H. Scrotal pain: evaluation and management. Korean J Urol 2015;56(01):3-11

4 Yu Y, Zhang F, An Q, Wang L, Li C, Xu Z. Scrotal Exploration for Testicular Torsion and Testicular Appendage Torsion: Emergency and Reality. Iran J Pediatr 2015;25(01):e248 
5 Beni-Israel T, Goldman M, Bar Chaim S, Kozer E. Clinical predictors for testicular torsion as seen in the pediatric ED. Am J Emerg Med 2010;28(07):786-789

6 Boettcher M, Bergholz R, Krebs TF, Wenke K, Aronson DC. Clinical predictors of testicular torsion in children. Urology 2012;79(03): 670-674

7 Sharp VJ, Kieran K, Arlen AM. Testicular torsion: diagnosis, evaluation, and management. Am Fam Physician 2013;88(12): 835-840

8 Yin S, Trainor JL. Diagnosis and Management of Testicular Torsion, Torsion of the Appendix Testis, and Epididymitis. Clin Pediatr Emerg Med 2009;10(01):38-44

9 Srinivasan A, Cinman N, Feber KM, Gitlin J, Palmer LS. History and physical examination findings predictive of testicular torsion: an attempt to promote clinical diagnosis by house staff. J Pediatr Urol $2011 ; 7(04): 470-474$
10 Rouzrokh M, Mirshemirani A, Khaleghnejad-Tabari A. Outcomes of Second Look Exploration in Testicular Torsion of Children. Iran J Pediatr 2015;25(04):e528

11 Dakhil S, Daraji F. How to Reduce Time Delays in Presentation and Treatment of Testicular Torsion; the Role of Public and Practitioners Education. Iraqi Postgrad Med J 2014;13(04):499-503

12 Ramachandra P, Palazzi K, Holmes N, Marietti S. Factors Influencing Rate of Testicular Salvage in Acute Testicular Torsion at a Tertiary Pediatric Center. Berkeley Plan J 2013;26(01):217-220

13 Lavallee ME, Cash J. Testicular torsion: evaluation and management. Curr Sports Med Rep 2005;4(02):102-104

14 Fabiani A, Calabrese M, Filosa A, et al. Explorative surgery for acute scrotal pain: The importance of patient age, side affected, time to surgery and surgeon. Arch Ital Urol Androl 2016;88(03):189-194

15 Sigalos JT, Pastuszak AW. Chronic orchialgia: epidemiology, diagnosis and evaluation. Transl Androl Urol 2017;6(Suppl 1):S37-S43 